\title{
Viscosity solutions of nonlinear integro-differential equations
}

by

Olivier ALVAREZ

and

Agnès TOURIN

CEREMADE, Université Paris IX-Dauphine, Place de Lattre de Tassigny, 75775 Paris Cedex 16, France.

ABSTRACT. - We investigate the questions of the existence and uniqueness of viscosity solutions to the Cauchy problem for integro-differential PDEs with nonlinear integral term. The existence of a solution is established by considering semicontinuous subsolutions and supersolutions and applying Perron's method. Uniqueness is proved for both bounded and unbounded solutions. These results are then applied to a problem arising in Finance, namely the stochastic differential utility model under mixed PoissonBrownian information.

Key words: viscosity solutions, nonlinear integro-differential parabolic PDE, stochastic differential utility.

RÉSUMÉ. - Nous étudions l'existence et l'unicité de solutions de viscosité du problème de Cauchy pour des équations aux dérivées partielles integrodifférentielles dont le terme intégral est non linéaire. L'existence est obtenue par la méthode de Perron et des principes de comparaison sont prouvés pour des fonctions bornées ou non bornées. Enfin, nous appliquons ces résultats au modèle économique d'utilité différentielle stochastique adaptée à la filtration engendrée par un mouvement Brownien et un processus de Poisson.

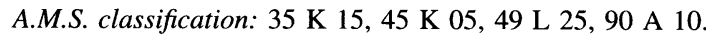




\section{INTRODUCTION}

In this paper, we study the existence and uniqueness of a solution to the Cauchy problem for a nonlinear integro-differential equation of the form

$$
\begin{aligned}
& -\partial_{t} u(t, x)+F\left(t, x, u(t, x), D u(t, x), D^{2} u(t, x)\right) \\
& -\int M(u(t, x+z), u(t, x)) m_{t, x}(d z)=0 \text { on }(0, T) \times \mathbb{R}^{N} ; \\
u(T, x) & =u_{T}(x) \text { on } \mathbb{R}^{N},
\end{aligned}
$$

where $F \in C\left((0, T] \times \mathbb{R}^{N} \times \mathbb{R} \times \mathbb{R}^{N} \times \mathcal{S}_{N}\right), M \in C(\mathbb{R} \times \mathbb{R}), m_{t, x}$ is a bounded positive measure, $u_{T} \in C\left(\mathbb{R}^{N}\right), D u$ denotes the space gradient and $D^{2} u$ the matrix of space second derivatives.

We propose an extension to (1) of the notion of viscosity solutions, whose original theory applies to fully nonlinear pössibly degenerate partial differential equations - we refer to the User's guide to viscosity solutions [3] for a presentation of the theory. In addition to the classical requirements that $F$ be elliptic:

$$
F(t, x, a, q, A) \geq F(t, x, a, q, B) \text { when } A \leq B,
$$

and satisfy, for some $\lambda \in \mathbb{R}$,

$$
F(t, x, b, q, A)-F(t, x, a, q, A) \geq \lambda(b-a) \text { when } a \leq b,
$$

we shall essentially impose that $M$ is nondecreasing with respect to the first variable:

$$
M(a, c) \leq M(b, c) \text { when } a \leq b .
$$

Most works about viscosity solutions of integro-differential equations we are aware of treat the linear case $(M(a, b)=a-b)$ and the related optimal control problem (with an integral term of the form $\left.\sup _{\alpha} \int u(t, x+z)-u(t, x) m_{t, x}^{\alpha}(d z)\right)$ and restrict to bounded continuous subsolutions and supersolutions (see for instance Soner [11]). In this paper, we allow the functions to be semicontinuous and unbounded (see Sayah [10] for results in this direction). This extension presents the great advantage of providing easily a solution via Perron's method under very general assumptions. In addition, a difficulty arises from the nonlinearity of the integral term for proving a comparison principle. Indeed, unlike the linear 
case where comparison follows at once from the observation that, at a maximum point $(t, x)$ of $u-v: u(t, x+z)-u(t, x) \leq v(t, x+z)-v(t, x)$ for all $z$, a general $M$ does not offer such monotonicity, even under (3).

To simplify the presentation, we shall focus on linear $F$, but comparison results could be obtained for more general local operators as in Crandall, Ishii and Lions [3]. And we shall apply these methods to the following equation arising from the stochastic differential utility model under PoissonBrownian information, in Finance theory

$$
\begin{aligned}
& -\partial_{t} u-\frac{1}{2} \operatorname{tr} a D^{2} u-\frac{1}{2} M_{11}(u, u)\langle a D u, D u\rangle-\langle b, D u\rangle-g(t, x, u) \\
& -\int M(u(t, x+z), u(t, x)) m_{t, x}(d z)=0 \text { on }(0, T) \times \mathbb{R}^{N} \\
u(T, x) & =u_{T}(x) \text { on } \mathbb{R}^{N} .
\end{aligned}
$$

We note here that the underlying probabilistic problem was solved by Ma [8], using the theory of stochastic differential equations. But the results we obtain for (4) remain of interest, since the exclusive use of PDE arguments allows to weaken most assumptions (concerning the measure $m$, the regularity of $M$, or the case of unbounded solutions).

In order not to obscure the main ideas of this work, we leave to the reader very natural extensions appearing in the literature, which can be treated within the framework we propose here. For instance, we could allow $M$ to depend on $t, x$, consider the associated optimal control problem, allow more general jumps with size $\gamma(t, x, z)$ instead of $z$, as well as unbounded measures (in the neighbourhood of 0) - as in Bensoussan and Lions [2], Sayah [10], or Soner [12].

The paper is organized as follows. In section 2 , we give equivalent definitions of semicontinuous subsolutions and supersolutions of (1). We then establish a version of Perron's method, adapted to the Cauchy problem. Section 3 is devoted to comparison principles for the equation with a linear local part $\left(M_{11}(a, a) \equiv 0\right.$ in (4)). After obtaining a preliminary restrictive result for bounded functions, we prove comparison for general $M$ and unbounded functions. The last section relates (4) to its stochastic interpretation and uses the change of variable introduced by Duffie and Lions [5] to obtain general existence and uniqueness results.

We close the Introduction with a few notations and conventions. Most come directly from the theory of viscosity solutions we assume the reader to be familiar with. Further information can be found in the User's guide [3]. Given an open subset $\Omega$ of $\mathbb{R}^{M}$, we denote by $C_{b}(\Omega)$ 
the set of the bounded continuous functions in $\Omega$, and $C_{c}(\Omega)$ the set of the continuous functions with compact support in $\Omega . B_{r}(x)$ is the open ball of $\mathbb{R}^{M}$ centered at $x$ with radius $r$. As a general rule, we associate with $(0, T] \times \mathbb{R}^{N}$ its relative topology; for clarity however, we shall denote $B_{r}^{+}(t, x)=B_{r}(t, x) \cap\left((0, T] \times \mathbb{R}^{N}\right)$. With a locally bounded function in $(0, T] \times \mathbb{R}^{N}$, we associate its upper semicontinuous envelope $u^{\star}(t, x)=\lim \sup _{(0, T] \times \mathbb{R}^{N} \ni(s, y) \rightarrow(t, x)} u(s, y)$ (and, respectively, its lower semicontinuous envelope $\left.u_{\star}(t, x)=-(-u)^{\star}(t, x)\right)$. Given $u \in U S C\left((0, T] \times \mathbb{R}^{N}\right)$ and $t, x \in(0, T] \times \mathbb{R}^{N}$, we define the parabolic superjet:

$$
\begin{aligned}
\mathcal{P}^{2,+} u(t, x)=\{ & (p, q, A) \in \mathbb{R} \times \mathbb{R}^{N} \times \mathcal{S}_{N} / u(s, y) \leq u(t, x)+p(s-t) \\
& +\langle q, y-x\rangle+\frac{1}{2}\langle A(y-x), y-x\rangle \\
& \left.+o\left(|s-t|+|y-x|^{2}\right) \text { as }(s, y) \rightarrow(t, x)\right\}
\end{aligned}
$$

as well as its closure:

$$
\begin{aligned}
\overline{\mathcal{P}}^{2,+} u(t, x)=\{ & (p, q, A) \\
& =\lim _{n \rightarrow \infty}\left(p_{n}, q_{n}, A_{n}\right) \text { with }\left(p_{n}, q_{n}, A_{n}\right) \in \mathcal{P}^{2,+} u\left(t_{n}, x_{n}\right) \\
& \text { and } \left.\lim _{n \rightarrow \infty}\left(t_{n}, x_{n}, u\left(t_{n}, x_{n}\right)\right)=(t, x, u(t, x))\right\} .
\end{aligned}
$$

If $u \in L S C\left((0, T] \times \mathbb{R}^{N}\right)$, we consider its subjet $\mathcal{P}^{2,-} u(t, x)=$ $-\mathcal{P}^{2,+}(-u)(t, x)$ and its closure $\overline{\mathcal{P}}^{2,-} u(t, x)=-\overline{\mathcal{P}}^{2,+}(-u)(t, x)$. Note that the semijets are defined at the boundary $\{t=T\}$, for technical reasons.

\section{VISCOSITY SOLUTIONS AND PERRON'S METHOD}

In this section, we extend the definition of semicontinuous viscosity solutions to integro-differential equation (1). As usual, we give an intrinsic definition (depending only on the functions and on their semijets) as well as an equivalent one that involves test functions. We also show that Perron's method still works for (1): the existence programme then amounts to establishing a comparison principle and finding a subsolution and a supersolution. As most of the results in this section use the classical viscosity solution techniques, we shall insist on the treatment of the integral term and sketch the remaining arguments. 
First of all, we have to make sure that the integral term is well defined and has some regularity. Consequently, we assume at least that

for every $(t, x) \in(0, T] \times \mathbb{R}^{N}, m_{t, x}$ is a bounded positive measure on $\mathbb{R}^{N}$

and $\lim _{s, y \rightarrow t, x} \int h(z) m_{s, y}(d z)=\int h(z) m_{t, x}(d z)$ provided $h \in C_{c}\left(\mathbb{R}^{N}\right)$.

Because we wish to consider unbounded solutions, we must strengthen the preceding requirement that $m$ be a bounded measure. We shall assume that, for every $(t, x) \in(0, T] \times \mathbb{R}^{N}$, there is some nonnegative continuous $\Phi$ satisfying

$$
\int \Phi(z) m_{t, x}(d z)<\infty \text { and } \lim _{s, y \rightarrow t, x} \int \Phi(z) m_{s, y}(d z)=\int \Phi(z) m_{t, x}(d z)
$$

The next lemma clarifies how (5) and (6) can be combined. First, it states that (5) is still true for continuous functions that are bounded by $\Phi$. (This extends the well-known observation that when $m$ is a probability measure $\left(m_{t, x}\left(\mathbb{R}^{N}\right) \equiv 1\right)$, (5) holds for functions in $C_{b}\left(\mathbb{R}^{N}\right)$.) Second, it says that, if $h$ is semicontinuous, then the integral term has the same semicontinuity (as a function of $(t, x)$ ).

Lemma 1. - Let $m$ satisfy (5) and $\varphi \in U S C\left((0, T] \times \mathbb{R}^{N} \times \mathbb{R}^{N}\right)$ be locally bounded. Assume that for some $(t, x) \in(0, T] \times \mathbb{R}^{N}$, there are $\eta>0$ and some nonnegative $\Phi \in C\left(\mathbb{R}^{N}\right)$ satisfying (6) such that

$$
\varphi(s, y, z) \leq \Phi(z) \text { for } s, y, z \in B_{\eta}^{+}(t, x) \times \mathbb{R}^{N}
$$

Then

$$
\limsup _{s, y \rightarrow t, x} \int \varphi(s, y, z) m_{s, y}(d z) \leq \int \varphi(t, x, z) m_{t, x}(d z)
$$

Remark 1. - For later use, we note that the preceding lemma implies that if $m$ satisfies (5) and if, for every $(t, x) \in(0, T] \times \mathbb{R}^{N}$, there is some continuous $\Phi \geq 1$ satisfying (6), then $(t, x) \mapsto m_{t, x}\left(\mathbb{R}^{N}\right)$ is continuous.

Proof. - We first assume that $\varphi$ has compact support in $(0, T] \times \mathbb{R}^{N} \times \mathbb{R}^{N}$. We can find $\epsilon>0$, a nonnegative function $\chi \in C_{c}\left(\mathbb{R}^{N}\right)$ such that $\chi \equiv 1$ on $B_{1 / \epsilon}(0)$ and a nonincreasing sequence of $\phi_{n} \in C_{c}\left([\epsilon, T] \times B_{1 / \epsilon}(0) \times\right.$ 
$\left.B_{1 / \epsilon}(0)\right)$ such that $\phi_{n} \downarrow \varphi$. From (5), we deduce that, for every $n$,

$$
\begin{aligned}
& \limsup _{s, y \rightarrow t, x} \int \varphi(s, y, z) m_{s, y}(d z) \leq \limsup _{s, y \rightarrow t, x} \int \phi_{n}(s, y, z) m_{s, y}(d z) \\
& \leq \limsup _{s, y \rightarrow t, x} \int\left\{\phi_{n}(t, x, z)\right. \\
& \left.\quad+\chi(z) \sup _{z \in B_{1 / \epsilon}(0)}\left|\phi_{n}(s, y, z)-\phi_{n}(t, x, z)\right|\right\} m_{s, y}(d z) \\
& =\int \phi_{n}(t, x, z) m_{t, x}(d z) .
\end{aligned}
$$

Sending $n \rightarrow \infty$, we conclude by Lebesgue's monotone convergence theorem that $\limsup _{s, y \rightarrow t, x} \int \varphi(s, y, z) m_{s, y}(d z) \leq \int \varphi(t, x, z) m_{t, x}(d z)$.

When the support of $\varphi$ is no longer compact, we construct, for $n$ large enough, $\chi_{n} \in C_{c}\left(\left(\frac{1}{n+1}, T\right] \times B_{n+1}(0) \times B_{n+1}(0)\right)$ nonnegative such that $\chi_{n} \equiv 1$ on $\left[\frac{1}{n}, T\right] \times \bar{B}_{n}(0) \times \bar{B}_{n}(0)$. For $(s, y) \in B_{\eta}^{+}(t, x)$, one has

$$
\begin{aligned}
& \int \varphi(s, y, z) m_{s, y}(d z) \\
& \quad \leq \int \chi_{n}(s, y, z) \varphi(s, y, z)+\left(1-\chi_{n}(s, y, z)\right) \Phi(z) m_{s, y}(d z) .
\end{aligned}
$$

By the preceding paragraph, as $(s, y) \rightarrow(t, x)$, the upper limit of the first expression in the right-hand term is smaller than $\int \chi_{n}(t, x, z) \varphi(t, x, z) m_{t, x}(d z)$, while $\int \chi_{n}(s, y, z) \Phi(z) m_{s, y}(d z) \rightarrow$ $\int \chi_{n}(t, x, z) \Phi(z) m_{t, x}(d z)$. From (6), we deduce that

$\limsup _{s, y \rightarrow t, x} \int \varphi(s, y, z) m_{s, y}(d z) \leq \int \chi_{n} \varphi(t, x, z)+\left(1-\chi_{n}\right) \Phi(z) m_{t, x}(d z)$.

Sending $n \rightarrow \infty$, we use Fatou's lemma and obtain the inequality we claimed.

The preceding lemma is the motivation for the following requirement on upper semicontinous functions that we shall be considering:

for every $(t, x) \in(0, T] \times \mathbb{R}^{N}$, there are $\Phi \in C\left(\mathbb{R}^{N}\right), \Phi \geq 1$ satisfying (6) and $\eta>0$ such that, for

$$
s, y, z \in B_{\eta}^{+}(t, x) \times \mathbb{R}^{N}, M(u(s, y+z), u(s, y)) \leq \Phi(z) .
$$

Since $M$ is nondecreasing, observe that, if $\left(t_{n}, x_{n}, u\left(t_{n}, x_{n}\right)\right) \rightarrow$ $(t, x, u(t, x)),(7)$ and the proof of Lemma 1 imply that

$$
\begin{array}{r}
\limsup _{n \rightarrow \infty} \int M\left(u\left(t_{n}, x_{n}+z\right), u\left(t_{n}, x_{n}\right)\right) m_{t_{n}, x_{n}}(d z) \\
\leq \int M(u(t, x+z), u(t, x)) m_{t, x}(d z) .
\end{array}
$$


The fact that semicontinuity is preserved in the nonlinear integral term will be used extensively. Let us mention immediately that, in practice, the construction of $\Phi$ (depending on $(t, x)$ ) in (6) imposes very natural and simple conditions on $m$ and on the asymptotic behaviour of $u$ and $M$. For instance, if $u$ is locally bounded and bounded from above, we shall assume that $t, x \mapsto m_{t, x}\left(\mathbb{R}^{N}\right)$ is continuous and choose $\Phi \equiv \max \left(1, \sup _{B_{\eta}^{+}(t, x)} M\left(\left\|u^{+}\right\|_{\infty}, u(s, y)\right)\right)$. In a similar way, we require that a lower semicontinous function satisfy

for every $(t, x) \in(0, T] \times \mathbb{R}^{N}$, there are $\Phi \in C\left(\mathbb{R}^{N}\right), \Phi \geq 1$ satisfying (6) and $\eta>0$ such that, for

$$
s, y, z \in B_{\eta}^{+}(t, x) \times \mathbb{R}^{N}, M(u(s, y+z), u(s, y)) \geq-\Phi(z) .
$$

We can now define the notion of semicontinuous subsolutions and supersolutions of (1) which is a straightforward adaptation of [3].

Definition 1. - A locally bounded function $u \in U S C\left((0, T] \times \mathbb{R}^{N}\right)$ satisfying (7) is a viscosity subsolution of (1) if, for all $x \in \mathbb{R}^{N}$, $u(T, x) \leq u_{T}(x)$ and, for all $(t, x) \in(0, T) \times \mathbb{R}^{N},(p, q, A) \in \mathcal{P}^{2,+} u(t, x)$ :

$$
-p+F(t, x, u(t, x), q, A)-\int M(u(t, x+z), u(t, x)) m_{t, x}(d z) \leq 0 .
$$

A locally bounded function $u \in L S C\left((0, T] \times \mathbb{R}^{N}\right)$ satisfying (8) is a viscosity supersolution of (1) if, for all $x \in \mathbb{R}^{N}, u(T, x) \geq u_{T}(x)$ and, for all $(t, x) \in(0, T) \times \mathbb{R}^{N},(p, q, A) \in \mathcal{P}^{2,-} u(t, x)$ :

$$
-p+F(t, x, u(t, x), q, A)-\int M(u(t, x+z), u(t, x)) m_{t, x}(d z) \geq 0 .
$$

$u \in C\left((0, T] \times \mathbb{R}^{N}\right)$, satisfying (7) and (8), is a viscosity solution of (1) if it is a viscosity subsolution and a viscosity supersolution of (1).

Remark 2. - It is equivalent to require that a subsolution (resp. supersolution) satisfy (9) (resp. (10)) for $(p, q, A) \in$ $\overline{\mathcal{P}}^{2,+} u(t, x) \quad\left(\right.$ resp. $\quad \overline{\mathcal{P}}^{2,-} u(t, x)$ ). Indeed, consider a sequence $\left(t_{n}, x_{n}\right) \in(0, T) \times \mathbb{R}^{N}$ as well as $\left(p_{n}, q_{n}, A_{n}\right) \in \mathcal{P}^{2,+} u\left(t_{n}, x_{n}\right)$ with $\lim _{n \rightarrow \infty}\left(t_{n}, x_{n}, u\left(t_{n}, x_{n}\right), p_{n}, q_{n}, A_{n}\right)=(t, x, u(t, x), p, q, A)$; taking the upper limit in (9) (written at $\left(t_{n}, x_{n}\right)$ ) as $n \rightarrow \infty$, and using the upper semicontinuity of the integral term as well as the continuity of $F$, we conclude that (9) holds at $(t, x)$ for $(p, q, A)$. An analogous statement is true for supersolutions.

This remark will be particularly useful for establishing uniqueness for (1). The existence programme needs the following equivalent definition. 
DEFINITION 2 (Equivalent). - A locally bounded function $u \in$ $U S C\left((0, T] \times \mathbb{R}^{N}\right)$ satisfying (7) is a viscosity subsolution of (1) if, for all $x \in \mathbb{R}^{N}, u(T, x) \leq u_{T}(x)$ and, for all $(t, x) \in(0, T) \times \mathbb{R}^{N}, \phi \in$ $C^{2}\left((0, T] \times \mathbb{R}^{N}\right)$ such that $u(t, x)=\phi(t, x), u<\phi$ on $(0, T] \times \mathbb{R}^{N} /(t, x)$, we have:

$$
-\partial_{t} \phi+F\left(t, x, \phi, D \phi, D^{2} \phi\right)-\int M(\phi(t, x+z), \phi(t, x)) m_{t, x}(d z) \leq 0 .
$$

A locally bounded function $u \in L S C\left((0, T] \times \mathbb{R}^{N}\right)$ satisfying (8) is a viscosity supersolution of (1) if, for all $x \in \mathbb{R}^{N}, u(T, x) \geq u_{T}(x)$ and, for all $(t, x) \in(0, T) \times \mathbb{R}^{N}, \phi \in C^{2}\left((0, T] \times \mathbb{R}^{N}\right)$ such that $u(t, x)=\phi(t, x)$, $u>\phi$ on $(0, T] \times \mathbb{R}^{N} /(t, x)$, we have:

$$
-\partial_{t} \phi+F\left(t, x, \phi, D \phi, D^{2} \phi\right)-\int M(\phi(t, x+z), \phi(t, x)) m_{t, x}(d z) \geq 0
$$

We briefly justify the equivalence between the two definitions. Regarding the subsolution property, we first observe that Definition 1 at once implies Definition 2 because of the monotonicity of $M$ and the fact that $\left(\partial_{t} \phi, D \phi, D^{2} \phi\right) \in \mathcal{P}^{2,+} u(t, x)$. Conversely, since $M(u(t, x+z), u(t, x)) \leq$ $\Phi(z)$, one can use the upper semicontinuity of the left-hand term to find $\phi_{0} \in$ $C\left((0, T] \times \mathbb{R}^{N}\right)$ such that $u<\phi_{0}$ and $M\left(\phi_{0}(t, x+z), u(t, x)\right) \leq \Phi(z)+1$ - we leave the details to the reader. Next, given $(p, q, A) \in \widehat{\mathcal{P}^{2}+} u(t, x)$, we construct a nonincreasing sequence of functions $\phi_{n} \in C^{2}$ such that

$$
\left(\phi_{n}(t, x), \partial_{t} \phi_{n}(t, x), D \phi_{n}(t, x), D^{2} \phi_{n}(t, x)\right)=(u(t, x), p, q, A),
$$

$u<\phi_{n} \leq \phi_{0}$ on $(0, T] \times \mathbb{R}^{N} /(t, x)$ and $\phi_{n} \downarrow u$ on $(0, T] \times \mathbb{R}^{N}$. But (11) then reads

$$
\begin{aligned}
-p+F(t, x, u(t, x), q, A) & \leq \int M\left(\phi_{n}(t, x+z), u(t, x)\right) m_{t, x}(d z) \\
& \downarrow \int M(u(t, x+z), u(t, x)) m_{t, x}(d z)
\end{aligned}
$$

as $n \rightarrow \infty$, where we have used Lebesgue's monotone convergence theorem to take the limit. And (9) is shown, proving thus our claim.

We momentarily assume that we have established a generic comparison principle for functions that lie between a subsolution $\underline{u} \in C\left((0, T] \times \mathbb{R}^{N}\right)$ and a supersolution $\bar{v} \in C\left((0, T] \times \mathbb{R}^{N}\right)$. And we check that the powerful Perron's method introduced by Ishii [7] extends to integro-differential 
equations (following mainly his argument) so as to obtain a viscosity solution of (1) $u \in C\left((0, T] \times \mathbb{R}^{N}\right)$ with $\underline{u} \leq u \leq \bar{v}$. Taking advantage of a particular property of the Cauchy problem observed by Barles and Perthame [1], we are in fact able to improve the tractability of Perron's method by not requiring that $\underline{u}$ and $\bar{v}$ coincide at the boundary $\{t=T\}$. Of course we have to make sure that the solution satisfies integrability conditions (7) and (8) and therefore we ask that:

for every $(t, x) \in(0, T] \times \mathbb{R}^{N}$, there are $\Phi \in C\left(\mathbb{R}^{N}\right), \Phi \geq 1$ satisfying (6) and $\eta>0$ such that for $s, y, z \in B_{\eta}^{+}(t, x) \times \mathbb{R}^{N}$,

$$
\begin{aligned}
a \in[\underline{u}(s, y), \bar{v}(s, y)]-\Phi(z) & \leq M(\underline{u}(s, y+z), a) \\
& \leq M(\bar{v}(s, y+z), a) \leq \Phi(z) .
\end{aligned}
$$

Proposition 1. - Let $F \in C\left((0, T] \times \mathbb{R}^{N} \times \mathbb{R} \times \mathbb{R}^{N} \times S_{N}\right)$ be elliptic, $M \in C(\mathbb{R} \times \mathbb{R})$ satisfy (3), m satisfy (5) and $u_{T} \in C\left(\mathbb{R}^{N}\right)$. Assume that there are $\underline{u}, \bar{v} \in C\left((0, T] \times \mathbb{R}^{N}\right)$ respectively a viscosity subsolution and a supersolution of (1) such that $\underline{u} \leq \bar{v}$ on $(0, T] \times \mathbb{R}^{N}$ and (12) holds. Assume also that the following comparison principle holds: if $u \in U S C\left((0, T] \times \mathbb{R}^{N}\right)$ and $v \in L S C\left((0, T] \times \mathbb{R}^{N}\right)$ are respectively a subsolution and $a$ supersolution of (1), with $u \leq \bar{v}$ and $v \geq \underline{u}$, then $u \leq v$ on $(0, T] \times \mathbb{R}^{N}$.

Then there is a unique viscosity solution $u \in C\left((0, T] \times \mathbb{R}^{N}\right)$ satisfying (7) and (8) such that $\underline{u} \leq u \leq \bar{v}$ on $(0, T] \times \mathbb{R}^{N}$.

Proof. - The uniqueness statement follows readily from comparison. For every $(t, x) \in(0, T] \times \mathbb{R}^{N}$, we define

$$
\begin{aligned}
& v(t, x) \\
& \quad=\sup \left\{u(t, x) / u \text { is a subsolution of (1) with } u \leq \bar{v} \text { on }(0, T] \times \mathbb{R}^{N}\right\} .
\end{aligned}
$$

We first note that $\underline{u} \leq v_{\star}, v^{\star} \leq \bar{v}$, because of the continuity of $\underline{u}, \bar{v}$, and therefore (12) implies that integrability condition (7) (resp. (8)) is fulfilled for $v^{\star}$ (resp. $v_{\star}$ ). We shall show that $v^{\star}, v_{\star}$ are respectively a subsolution and a supersolution of (1). Once this is proved, comparison will imply that $v^{\star} \leq v_{\star}$ on $(0, T] \times \mathbb{R}^{N}$. The reverse inequality holding obviously, we shall first conclude that $v \in C\left((0, T] \times \mathbb{R}^{N}\right)$ and then that $v$ is a viscosity solution of (1), with $\underline{u} \leq v \leq \bar{v}$, as asserted. As a matter of fact, we begin by establishing that $v^{\star}$ (resp. $v_{\star}$ ) is a subsolution (resp. supersolution) of (1) with generalized boundary condition, that is to say with the inequality 
$u(T, x) \leq u_{T}(x)$ in Definition 1 (resp. $\left.u(T, x) \geq u_{T}(x)\right)$ relaxed to $-p+F(T, x, u(T, x), q, A)-\int M(u(T, x+z), u(T, x)) m_{T, x}(d z) \leq 0$,

for $(p, q, A) \in \mathcal{P}^{2,+} u(T, x)$, if $u(T, x)>u_{T}(x)$ $\left(-p+F(T, x, u(T, x), q, A)-\int M(u(T, x+z), u(T, x)) m_{T, x}(d z) \geq 0\right.$,

for $(p, q, A) \in \mathcal{P}^{2,-} u(T, x)$, if $u(T, x)<u_{T}(x)$, respectively). We conclude by observing that a subsolution (resp. supersolution) to the Cauchy problem with generalized boundary condition is indeed a subsolution (resp. supersolution) in the sense of Definition 1, reproducing here the proof given by $[1$, Proposition 5], for the sake of completeness.

We first prove that $v^{\star}$ is a subsolution of (1) with generalized boundary condition. Let $(t, x) \in(0, T] \times \mathbb{R}^{N}$ and consider a sequence $s_{n}, y_{n}, u_{n}$ such that $\lim _{n \rightarrow \infty}\left(s_{n}, y_{n}, u_{n}\left(s_{n}, y_{n}\right)\right)=\left(t, x, v^{\star}(t, x)\right), u_{n}$ being a subsolution of (1) for every $n$. If $\phi \in C^{2}$ is such that $v^{\star}(t, x)=\phi(t, x)$ and $v^{\star}<\phi$ on $(0, T] \times \mathbb{R}^{N} /(t, x)$, there is a sequence of local maxima $\left(t_{n}, x_{n}\right)$ of $u_{n}-\phi$ (for $n$ large enough) such that $\lim _{n \rightarrow \infty}\left(t_{n}, x_{n}, u_{n}\left(t_{n}, x_{n}\right)\right)=$ $\left(t, x, v^{\star}(t, x)\right)$. In addition, $t_{n}<T$ for large $n$, unless $t=T$ and $v^{\star}(T, x) \leq u_{T}(x)$, for $u_{T}$ is continuous. But, when $t_{n}<T$, one has

$$
\begin{aligned}
& -\partial_{t} \phi+F\left(t_{n}, x_{n}, u_{n}, D \phi, D^{2} \phi\right) \\
\leq & \int M\left(\phi\left(t_{n}, x_{n}+z\right), u_{n}\left(t_{n}, x_{n}\right)\right) m_{t_{n}, x_{n}}(d z),
\end{aligned}
$$

where we have used (3) together with the inequality $u_{n} \leq \phi$; letting $n \rightarrow \infty$ and invoking Lemma 1, we conclude, as claimed, that

$$
-\partial_{t} \phi+F\left(t, x, v^{\star}, D \phi, D^{2} \phi\right) \leq \int M\left(\phi(t, x+z), v^{\star}(t, x)\right) m_{t, x}(d z) .
$$

We now assume that $v_{\star}$ is not a supersolution of (1) with generalized boundary condition, so that there is $(t, x) \in(0, T] \times \mathbb{R}^{N}$ and $\phi \in C^{2}$ with $v_{\star}(t, x)=\phi(t, x), v_{\star}>\phi$ on $(0, T] \times \mathbb{R}^{N} /(t, x), v_{\star}(T, x)<u_{T}(x)$ if $t=T$ and

$$
\begin{aligned}
& -\partial_{t} \phi+F\left(t, x, \phi, D \phi, D^{2} \phi\right) \\
< & \int M(\max (\phi(t, x+z), \underline{u}(t, x+z)), \phi(t, x)) m_{t, x}(d z) .
\end{aligned}
$$


First, we observe that $v_{\star}(t, x)<\bar{v}(t, x)$. Indeed, $v_{\star}(t, x)=\bar{v}(t, x)$ would imply that $\left(\partial_{t} \phi(t, x), D \phi(t, x), D^{2} \phi(t, x)\right) \in \mathcal{P}^{2,-} \bar{v}(t, x)$; but the fact that $\bar{v}$ is a supersolution contradicts (13). Therefore, for $\eta_{1}, \delta_{1}>0$ small enough, $\phi+\delta_{1} \leq \bar{v}$ on $B_{\eta_{1}}^{+}(t, x)$ and $\phi(T, y)+\delta_{1} \leq u_{T}(y)$ if $(T, y) \in B_{\eta_{1}}^{+}(t, x) \cap\{t=T\}$. The continuity of the integral term (Lemma 1 and (12)) provides $\eta_{2}, \delta_{2}>0$ such that

$$
\begin{aligned}
&-\partial_{t} \phi+F\left(s, y, \phi+\delta, D \phi, D^{2} \phi\right) \\
& \leq \int M(\max (\phi(s, y+z)+\delta, \underline{u}(s, y+z)), \\
&\max (\phi(s, y)+\delta, \underline{u}(s, y))) m_{s, y}(d z),
\end{aligned}
$$

for all $s, y, \delta \in B_{\eta_{2}}^{+}(t, x) \times\left[0, \delta_{2}\right]$. Lastly, we obtain $\delta_{3}>0$ for which $v_{\star}>$ $\phi+\delta_{3}$ on $\partial B_{\eta_{0}}^{+}(t, x)$ for $\eta_{0}=\min \left(\eta_{1}, \eta_{2}\right)$. Setting $\delta_{0}=\min \left(\delta_{1}, \delta_{2}, \delta_{3}\right)>0$, we define

$$
w=\max \left(v^{\star}, \phi+\delta_{0}\right) \text { on } B_{\eta_{0}}^{+}(t, x) ; w=v^{\star} \text { elsewhere. }
$$

The preceding construction guarantees that $w \in U S C\left((0, T] \times \mathbb{R}^{N}\right)$, $\underline{u} \leq w \leq \bar{v}$ on $(0, T] \times \mathbb{R}^{N}$. We claim that $w$ is a subsolution of (1) with generalized boundary condition. Indeed, let $(s, y) \in(0, T] \times \mathbb{R}^{N}$ and $\chi \in C^{2}$ be such that $w(s, y)=\chi(s, y)$ and $w<\chi$ on $(0, T] \times \mathbb{R}^{N} /(s, y)$. Then, it is clear that, whether $w(s, y)=v^{\star}(s, y)$ or not, $\left(\partial_{t} \chi(s, y), D \chi(s, y), D^{2} \chi(s, y)\right)$ belongs to $\mathcal{P}^{2,+} v^{\star}(s, y)$ or $\mathcal{P}^{2,+}(\phi+$ $\left.\delta_{0}\right)(s, y)$. The fact that $v^{\star}$ is a subsolution and (14) then imply (9). Finally, observe that $w_{\star}(t, x) \geq \max \left(v_{\star}(t, x), \phi(t, x)+\delta_{0}\right)>v_{\star}(t, x)$; hence there is $(s, y)$ such that $w(s, y)>v(s, y)$. We thus obtain a contradiction with the definition of $v$ once we know that $w$, as a subsolution of (1) with generalized boundary condition, satisfy $w(T, x) \leq u_{T}(x)$ on $\mathbb{R}^{N}$. We now prove this inequality.

So, let $u \in U S C\left((0, T] \times \mathbb{R}^{N}\right)$ be a subsolution of (1) with generalized boundary condition and suppose that $u(T, x)>u_{T}(x)$ for some $x \in \mathbb{R}^{N}$. Fix $\eta>0$ so small that $M(u(s, y+z), u(s, y)) \leq \Phi(z)$, for $s, y, z \in$ $B_{\eta}^{+}(T, x) \times \mathbb{R}^{N}$, and $u(T, x)>u_{T}(y)$, for $y \in B_{\eta}(x)$. For $\epsilon>0$, we choose $C_{\epsilon} \rightarrow \infty$ as $\epsilon \rightarrow 0$ such that

$$
C_{\epsilon}>\sup _{B_{\eta}^{+}(T, x)}\left\{-F\left(s, y, u(s, y), \frac{2(y-x)}{\epsilon}, \frac{2 I}{\epsilon}\right)+\int \Phi(z) m_{s, y}(d z)\right\},
$$

and consider $\left(t_{\epsilon}, x_{\epsilon}\right)$ a maximum point of $u(s, y)-\frac{|y-x|^{2}}{\epsilon}-C_{\epsilon}(T-s)$ on $B_{\eta}^{+}(T, x)$. Since $\lim _{\epsilon \rightarrow 0}\left|x_{\epsilon}-x\right|=0, \lim _{\epsilon \rightarrow 0}\left|t_{\epsilon}-T\right|=0$ and 
$u\left(T, x_{\epsilon}\right) \geq u(T, x)>u_{T}\left(x_{\epsilon}\right)$ when $t_{\epsilon}=T$, we conclude that (9) should hold at $\left(-C_{\epsilon}, \frac{2\left(x_{\epsilon}-x\right)}{\epsilon}, \frac{2 I}{\epsilon}\right) \in \mathcal{P}^{2,+} u_{\epsilon}\left(t_{\epsilon}, x_{\epsilon}\right)$, for $\epsilon$ small enough, which would contradict our choice of $C_{\epsilon}$. The argument for the supersolution proceeds exactly in the same way.

\section{COMPARISON RESULTS}

We now turn to establishing comparison principles. As mentioned in the Introduction, we begin by considering the version of (1) with a linear local term

$$
\begin{aligned}
& -\partial_{t} u(t, x)-\frac{1}{2} \operatorname{tr} a(t, x) D^{2} u(t, x) \\
& -\langle b(t, x), D u(t, x)\rangle-g(t, x, u(t, x)) \\
& -\int M(u(t, x+z), u(t, x)) m_{t, x}(d z)=0 \text { on }(0, T) \times \mathbb{R}^{N} ; \\
u(T, x) & =u_{T}(x) \text { on } \mathbb{R}^{N},
\end{aligned}
$$

and we assume that

$$
a=\sigma \sigma^{T} \text { and }|\sigma(t, x)-\sigma(t, y)|+|b(t, x)-b(t, y)| \leq C|x-y|,
$$

the standard hypothesis for comparison to hold for viscosity solutions of linear partial differential equations. At first, we impose the restrictive conditions

$$
\begin{gathered}
g(t, x, b)-g(t, x, a) \leq a-b \text { when } a \leq b, \\
M(a+h, b+h)-M(a, b) \leq 0 \text { when } h \geq 0,
\end{gathered}
$$

which actually guarantee comparison for bounded subsolutions and supersolutions of the more delicate stationary problem. But it is wellknown, in the case of the Cauchy problem with no integral term, how requirement (17) can be relaxed to a Lipschitz regularity in a (uniformly in $(t, x))$ - this corresponds to the observation that, in (2), the requirement $\lambda>0$ for the stationary problem can be relaxed to $\lambda \in \mathbb{R}$. More precisely, we shall prove that the comparison principle holds for bounded functions when $g$ and $M$ are locally Lipschitz; and, provided they are globally Lipschitz, we shall allow the functions to be compared to have an exponential or polynomial growth, depending on whether $\sigma$ and $b$ are bounded or not. 
Considering first bounded from above (resp. below) subsolutions (resp. supersolutions), we require that

$$
t, x \mapsto m_{t, x}\left(\mathbb{R}^{N}\right) \text { is continuous and } m_{t, x}\left(\mathbb{R}^{N}\right) \leq C,
$$

which, as said before, imply integrability condition (7) (resp. (8)). We can now state the following comparison principle for bounded functions, whose proof, for the most part, mimics the classical argument of [3] for viscosity solutions of nonlinear partial differential equations. We just mention that, for technical reasons, we have to allow $M$ to depend on $t$ (an extension that presents no difficulty but involves some more cumbersome expressions), and thus we assume (18) to hold uniformly for $t \in(0, T]$.

Proposition 2. - Let $a, b \in C\left((0, T] \times \mathbb{R}^{N}\right)$ satisfy (16), $g \in$ $C\left((0, T] \times \mathbb{R}^{N} \times \mathbb{R}\right)$ satisfy $(17), M \in C((0, T] \times \mathbb{R} \times \mathbb{R})$ satisfy (3) and (18), $m$ satisfy (5) and (19), and $u_{T} \in C\left(\mathbb{R}^{N}\right)$. Let $u \in U S C\left((0, T] \times \mathbb{R}^{N}\right)$ be a bounded from above viscosity subsolution of (15) and $v \in$ $L S C\left((0, T] \times \mathbb{R}^{N}\right)$ be a bounded from below viscosity supersolution of (15).

Then

$$
u \leq v \text { on }(0, T] \times \mathbb{R}^{N} .
$$

Proof. - We argue by contradiction and thus assume that $\sup _{(0, T] \times \mathbb{R}^{N}} u(t, x)-v(t, x)>0$. We fix $\eta>0$ small enough so that $N=\sup _{(0, T] \times \mathbb{R}^{N}} u(t, x)-v(t, x)-\frac{\eta}{t}>0$ and for $\delta>0$, we set

$$
N_{\delta}=\max _{(0, T] \times \mathbb{R}^{N}} u(t, x)-v(t, x)-\frac{\eta}{t}-\delta|x|^{2},
$$

which is positive for $\delta$ small enough. Following the general viscosity solution technique, we consider a global maximum point $\left(t_{\epsilon}, x_{\epsilon}, y_{\epsilon}\right) \in$ $(0, T] \times \mathbb{R}^{N} \times \mathbb{R}^{N}$ of $u(t, x)-v(t, y)-\frac{\eta}{t}-\delta|x|^{2}-\frac{|x-y|^{2}}{\epsilon}$. It is a classical and easy exercise (see [3, Lemma 3.1]) to check that, along a subsequence, $\lim _{\epsilon \rightarrow 0}\left(t_{\epsilon}, x_{\epsilon}, y_{\epsilon}\right)=\left(t_{\delta}, x_{\delta}, x_{\delta}\right)$, for some $\left(t_{\delta}, x_{\delta}\right) \in(0, T) \times \mathbb{R}^{N}$, a maximum point of $u(t, x)-v(t, x)-\frac{\eta}{t}-\delta|x|^{2}$, and that

$$
\lim _{\epsilon \rightarrow 0} u\left(t_{\epsilon}, x_{\epsilon}\right)=u\left(t_{\delta}, x_{\delta}\right) \text { and } \lim _{\epsilon \rightarrow 0} v\left(t_{\epsilon}, y_{\epsilon}\right)=v\left(t_{\delta}, x_{\delta}\right) .
$$

In addition, one has

$$
\lim _{\delta \rightarrow 0} N_{\delta}=N \text { and } \lim _{\delta \rightarrow 0} \delta\left|x_{\delta}\right|^{2}=0 .
$$

Theorem 8.3 of [3] then provides $\left(p_{\epsilon}, q_{\epsilon}, A_{\epsilon}, B_{\epsilon}\right) \in \mathbb{R} \times \mathbb{R}^{N} \times \mathcal{S}_{N} \times \mathcal{S}_{N}$ such that

$$
\left(p_{\epsilon}-\frac{\eta}{t_{\epsilon}^{2}}, q_{\epsilon}+2 \delta x_{\epsilon}, A_{\epsilon}+2 \delta I\right) \in \overline{\mathcal{P}}^{2,+} u\left(t_{\epsilon}, x_{\epsilon}\right),\left(p_{\epsilon}, q_{\epsilon}, B_{\epsilon}\right) \in \overline{\mathcal{P}}^{2,-} v\left(t_{\epsilon}, y_{\epsilon}\right)
$$


and, under (16),

$$
\lim _{\epsilon \rightarrow 0}\left\langle b\left(t_{\epsilon}, x_{\epsilon}\right)-b\left(t_{\epsilon}, y_{\epsilon}\right), q_{\epsilon}\right\rangle=0, \limsup _{\epsilon \rightarrow 0} \operatorname{tr} a\left(t_{\epsilon}, x_{\epsilon}\right) A_{\epsilon}-\operatorname{tr} a\left(t_{\epsilon}, y_{\epsilon}\right) B_{\epsilon} \leq 0 .
$$

The observation that $t_{\epsilon}<T$ for $\epsilon$ small enough and the fact that $u$ and $v$ are respectively a subsolution and a supersolution of (15) then yield

$$
\begin{aligned}
& -p_{\epsilon}+\frac{\eta}{t_{\epsilon}^{2}}-\frac{1}{2} \operatorname{tr} a\left(t_{\epsilon}, x_{\epsilon}\right) A_{\epsilon}-\delta \operatorname{tr} a\left(t_{\epsilon}, x_{\epsilon}\right) \\
& -\left\langle b\left(t_{\epsilon}, x_{\epsilon}\right), q_{\epsilon}\right\rangle-2 \delta\left\langle b\left(t_{\epsilon}, x_{\epsilon}\right), x_{\epsilon}\right\rangle \\
& -g\left(t_{\epsilon}, x_{\epsilon}, u\left(t_{\epsilon}, x_{\epsilon}\right)\right)-\int M\left(t_{\epsilon}, u\left(t_{\epsilon}, x_{\epsilon}+z\right), u\left(t_{\epsilon}, x_{\epsilon}\right)\right) m_{t_{\epsilon}, x_{\epsilon}}(d z) \leq 0
\end{aligned}
$$

and

$$
\begin{aligned}
& -p_{\epsilon}-\frac{1}{2} \operatorname{tr} a\left(t_{\epsilon}, y_{\epsilon}\right) B_{\epsilon}-\left\langle b\left(t_{\epsilon}, y_{\epsilon}\right), q_{\epsilon}\right\rangle-g\left(t_{\epsilon}, y_{\epsilon}, v\left(t_{\epsilon}, y_{\epsilon}\right)\right) \\
& -\int M\left(t_{\epsilon}, v\left(t_{\epsilon}, y_{\epsilon}+z\right), v\left(t_{\epsilon}, y_{\epsilon}\right)\right) m_{t_{\epsilon}, y_{\epsilon}}(d z) \geq 0 .
\end{aligned}
$$

We substract those two inequalities and take the upper limit as $\epsilon \rightarrow 0$, with Lemma 1 in mind, to conclude that

$$
\begin{aligned}
& -\delta \operatorname{tr} a\left(t_{\delta}, x_{\delta}\right)-2 \delta\left\langle b\left(t_{\delta}, x_{\delta}\right), x_{\delta}\right\rangle \\
& -g\left(t_{\delta}, x_{\delta}, u\left(t_{\delta}, x_{\delta}\right)\right)+g\left(t_{\delta}, x_{\delta}, v\left(t_{\delta}, x_{\delta}\right)\right) \\
\leq \int & \left\{M\left(t_{\delta}, u\left(t_{\delta}, x_{\delta}+z\right), u\left(t_{\delta}, x_{\delta}\right)\right)\right. \\
& \left.-M\left(t_{\delta}, v\left(t_{\delta}, x_{\delta}+z\right), v\left(t_{\delta}, x_{\delta}\right)\right)\right\} m_{t_{\delta}, x_{\delta}}(d z) \\
\leq \int & \left\{M\left(t_{\delta}, u\left(t_{\delta}, x_{\delta}+z\right)-u\left(t_{\delta}, x_{\delta}\right)+v\left(t_{\delta}, x_{\delta}\right), v\left(t_{\delta}, x_{\delta}\right)\right)\right. \\
- & \left.M\left(t_{\delta}, v\left(t_{\delta}, x_{\delta}+z\right), v\left(t_{\delta}, x_{\delta}\right)\right)\right\} m_{t_{\delta}, x_{\delta}}(d z),
\end{aligned}
$$

where the last inequality follows from (18) and the observation that $u\left(t_{\delta}, x_{\delta}\right) \geq v\left(t_{\delta}, x_{\delta}\right)$ for $\delta$ small. Remarking that, for $z \in \mathbb{R}^{N}$,

$$
u\left(t_{\delta}, x_{\delta}+z\right)-u\left(t_{\delta}, x_{\delta}\right)+v\left(t_{\delta}, x_{\delta}\right) \leq v\left(t_{\delta}, x_{\delta}+z\right)+N-N_{\delta},
$$

we use (19) and the continuity of $M$ to bound from above the last expression in (22) by $\omega_{R}\left(N-N_{\delta}\right) \sup _{(0, T] \times \mathbb{R}^{N}} m_{t, x}\left(\mathbb{R}^{N}\right)$ (for some $R$ depending on the 
upper bound of $u$ and the lower bound of $v$ ), which goes to zero as $\delta \rightarrow 0$. We also note that (16) implies that, for some $C>0$ independent of $\delta$,

$$
\operatorname{tr} a\left(t_{\delta}, x_{\delta}\right),\left\langle b\left(t_{\delta}, x_{\delta}\right), x_{\delta}\right\rangle \leq C\left(1+\left|x_{\delta}\right|^{2}\right) .
$$

Recalling (17) and (21), we send $\delta \rightarrow 0$ in (22) and conclude that

$$
\limsup _{\delta \rightarrow 0} u\left(t_{\delta}, x_{\delta}\right)-v\left(t_{\delta}, x_{\delta}\right) \leq 0,
$$

which contradicts our hypothesis that $N$ is positive.

Remark 3. - A careful inspection of the above proof shows that, instead of requiring (9) (resp. (10)) to hold at every $(t, x) \in(0, T) \times \mathbb{R}^{N}$, it just need be true when the set $\left\{y \in B_{1}(x) / u(t, x) \geq v(t, y)\right\}$ (resp. $\{y \in$ $\left.\left.B_{1}(x) / u(t, y) \geq v(t, x)\right\}\right)$ is not empty. Indeed, as $u\left(t_{\delta}, x_{\delta}\right)>v\left(t_{\delta}, x_{\delta}\right)$ for $\delta$ small enough, (20) implies that, for $\epsilon$ small enough, $u\left(t_{\epsilon}, x_{\epsilon}\right) \geq v\left(t_{\epsilon}, y_{\epsilon}\right)$ and $y_{\epsilon} \in B_{1}\left(x_{\epsilon}\right)$.

Remark 4. - We wish to mention that condition (19), which was used in an essential way, is optimal. Indeed, consider the linear equation

$$
-\partial_{t} u(t, x)-1-\int u(t, x+z)-u(t, x) m_{t, x}(d z)=0 \text { and } u(T, x)=0,
$$

which has $v(t, x)=T-t$ as a solution, whatever $m$ we choose. For $\alpha>0$ arbitrary, $C>0$, we define $m_{t, x}(d z)=C\left(1+|x|^{\alpha}\right) \delta_{x}(z)$ (with $\delta_{x}$ denoting the Dirac measure at $x)$. We claim that, for $C$ large, $u(t, x)=\frac{2(T-t)^{2}|x|^{\alpha}}{T\left(1+|x|^{\alpha}\right)}$ is a bounded subsolution of the equation, while comparison does not hold, for $\lim _{t \rightarrow 0,|x| \rightarrow \infty} u(t, x)-v(t, x)=T>0$. We first observe that $u(T, x)=0$ on $\mathbb{R}^{N}$ and we compute

$$
\begin{aligned}
& -\partial_{t} u(t, x)-1-\int u(t, x+z)-u(t, x) m_{t, x}(d z) \\
& =\frac{4(T-t)|x|^{\alpha}}{T\left(1+|x|^{\alpha}\right)}-1-\frac{C\left(2^{\alpha+1}-2\right)(T-t)^{2}|x|^{\alpha}}{T\left(1+2^{\alpha}|x|^{\alpha}\right)} \\
& \leq(T-t)^{2}\left\{\frac{4|x|^{2 \alpha}}{T^{2}\left(1+|x|^{\alpha}\right)^{2}}-\frac{C\left(2^{\alpha+1}-2\right)|x|^{\alpha}}{T\left(1+2^{\alpha}|x|^{\alpha}\right)}\right\} \leq 0
\end{aligned}
$$

for $C$ large.

We now relax (17) and (18) as follows: for $a, b, c, d \in[-R, R]$,

$$
\begin{aligned}
& g(t, x, b)-g(t, x, a) \leq C_{R}(b-a) \text { when } a \leq b, \\
& M(a, b)-M(c, d) \leq C_{R}(|a-c|+|b-d|),
\end{aligned}
$$


and obtain the following general comparison principle for bounded subsolutions and supersolutions.

Proposition 3. - Let $a, b \in C\left((0, T] \times \mathbb{R}^{N}\right)$ satisfy (16), $g \in$ $C\left((0, T] \times \mathbb{R}^{N} \times \mathbb{R}\right)$ satisfy (23), $M \in C(\mathbb{R} \times \mathbb{R})$ satisfy (3) and (24), $m$ satisfy (5) and (19), and $u_{T} \in C\left(\mathbb{R}^{N}\right)$. Let $u \in U S C\left((0, T] \times \mathbb{R}^{N}\right)$ be a bounded from above viscosity subsolution of (15) and $v \in$ $L S C\left((0, T] \times \mathbb{R}^{N}\right)$ be a bounded from below viscosity supersolution of (15).

Then

$$
u \leq v \text { on }(0, T] \times \mathbb{R}^{N} .
$$

Proof. - Setting $R=\max \left(\left\|u^{+}\right\|_{\infty},\left\|v^{-}\right\|_{\infty}\right)$, we define $M_{R}(a, b)=$ $M\left(\zeta_{R}(a), \zeta_{R}(b)\right)$ and $g_{R}(t, x, a)=g\left(t, x, \zeta_{R}(a)\right)$, with $\zeta_{R}(a)=$ $\min (\max (a,-R), R)$. We choose $C_{1}, C_{2}>0$ depending only on $R$ such that $g_{R}(t, x, b)-g_{R}(t, x, a) \leq C_{1}(b-a)$ when $b \geq a, M_{R}(a+h, b+h)-$ $M_{R}(a, b) \leq C_{2} h$ for $h \geq 0$ and $M(a, c) \leq M_{R}(a, c) \leq M_{R}(b, c) \leq M(b, c)$ for $a \leq b, a \leq R, b \geq-R$ and $c \in[-R, R]$. In particular, $u$ (resp. $v$ ) satisfies (9) (resp. (10)), with $g_{R}, M_{R}$ instead of $g, M$, at every $(t, x)$ such that $u(t, x) \geq-R$ (resp. $v(t, x) \leq R$ ) and thus when $u(t, x) \geq v(t, y)$ (resp. $u(t, y) \geq v(t, x)$ ) for some $y \in B_{1}(x)$.

For $K>0$ to be determined below, we set

$$
\begin{gathered}
g^{\prime}(t, x, a)=e^{K(t-T)} g_{R}\left(t, x, e^{K(T-t)} a\right)+\left(C_{2} m_{t, x}\left(\mathbb{R}^{N}\right)-K\right) a, \\
M^{\prime}(t, a, b)=e^{K(t-T)} M_{R}\left(e^{K(T-t)} a, e^{K(T-t)} b\right)-C_{2} b .
\end{gathered}
$$

A straightforward computation establishes that $u^{\prime}(t, x)=e^{K(t-T)} u(t, x)$ and $v^{\prime}(t, x)=e^{K(t-T)} v(t, x)$ are respectively a subsolution and a supersolution of (15) with $M^{\prime}, g^{\prime}$ instead of $M, g$ when $u^{\prime}(t, x) \geq v^{\prime}(t, y)$ (resp. $u^{\prime}(t, y) \geq v^{\prime}(t, x)$ ) for some $y \in B_{1}(x)$. Remark that $M^{\prime}$ satisfies (18), $g^{\prime}$ is continuous and for $a \leq b$

$$
g^{\prime}(t, x, b)-g^{\prime}(t, x, a) \leq\left(C_{1}+C_{2} \sup _{(0, T] \times \mathbb{R}^{N}} m_{t, x}\left(\mathbb{R}^{N}\right)-K\right)(b-a)=a-b,
$$

for the choice $K=1+C_{1}+C_{2} \sup _{(0, T] \times \mathbb{R}^{N}} m_{t, x}\left(\mathbb{R}^{N}\right)$. We apply Proposition 2 and Remark 3, and conclude that $u^{\prime} \leq v^{\prime}$ on $(0, T] \times \mathbb{R}^{N}$, which yields $u \leq v$.

Comparison for unbounded solutions requires the "local" regularity conditions (23) and (24) to be global, and thus we shall assume

$$
\begin{gathered}
g(t, x, b)-g(t, x, a) \leq C(b-a) \text { when } a \leq b, \\
M(a, b)-M(c, d) \leq C(|a-c|+|b-d|) .
\end{gathered}
$$


VISCOSITY SOLUTIONS OF NONLINEAR INTEGRO-DIFFERENTIAL EQUATIONS 309

We have to distinguish whether $\sigma$ and $b$ are bounded or

$$
|\sigma(t, x)|+|b(t, x)| \leq C(1+|x|)
$$

notice that this condition follows from (16) provided $\sigma(t, 0)$ and $b(t, 0)$ are bounded on $(0, T]$. In the first case, (19) is strengthened to

$t, x \mapsto \int e^{|z| /(\lambda+t)} m_{t, x}(d z)$ is continuous and $\int e^{|z| /(\lambda+t)} m_{t, x}(d z) \leq C$,

for some $\lambda>0$; this corresponds to the asymptotic behaviour for subsolutions and supersolutions

$$
\limsup _{|x| \rightarrow \infty} \sup _{(0, T]} u(t, x) e^{-|x| /(\lambda+t)} \leq 0, \liminf _{|x| \rightarrow \infty} \inf _{(0, T]} v(t, x) e^{-|x| /(\lambda+t)} \geq 0
$$

respectively. (Note that under (26) and (28), a function satisfying the first inequality in (29) fulfils the integrability condition (7) with $\Phi(z)=$ $C e^{|z| /(\lambda+t)}, C$ depending on $(t, x)$.) Our first unbounded comparison principle is the following:

Proposition 4. - Let $a, b \in C_{b}\left((0, T] \times \mathbb{R}^{N}\right)$ satisfy (16), $g \in$ $C\left((0, T] \times \mathbb{R}^{N} \times \mathbb{R}\right)$ satisfy (25), $M \in C(\mathbb{R} \times \mathbb{R})$ satisfy (3) and (26), $m$ satisfy (5) and (28), and $u_{T} \in C\left(\mathbb{R}^{N}\right)$. Let $u \in U S C\left((0, T] \times \mathbb{R}^{N}\right)$ be a viscosity subsolution of (15) and $v \in L S C\left((0, T] \times \mathbb{R}^{N}\right)$ be a viscosity supersolution of (15), that satisfy (29) respectively.

Then

$$
u \leq v \text { on }(0, T] \times \mathbb{R}^{N} .
$$

Proof. - Replacing, if necessary, $u$ (and $v$ ) by $u^{\prime}(t, x)=e^{K(t-T)} u(t, x)$ (and $v^{\prime}$ ), for some constant $K$ chosen as in the proof of Proposition 3, we assume, without loss of generality, that $g$ and $M$ satisfy respectively (17) and (18) (and we drop the dependence on $t$ of the new $M$ for convenience). For $C>0$, we set

$$
w(t, x)=\exp \frac{C+\sqrt{1+|x|^{2}}}{\lambda+t}
$$

and we compute

$$
-\partial_{t} w-\frac{1}{2} \operatorname{tr} a D^{2} w-\langle b, D w\rangle
$$




$$
\begin{aligned}
& -C_{M} \int|w(t, x+z)-w(t, x)| m_{t, x}(d z) \\
\geq & w(t, x)\left\{\frac{C+\sqrt{1+|x|^{2}}}{(\lambda+t)^{2}}\right. \\
& -\frac{\operatorname{tr} a(t, x)}{2(\lambda+t) \sqrt{1+|x|^{2}}}-\frac{\langle a(t, x) x, x\rangle}{2(\lambda+t)^{2}\left(1+|x|^{2}\right)} \\
& \left.-\frac{\langle b(t, x), x\rangle}{(\lambda+t) \sqrt{1+|x|^{2}}}-C_{M} \int e^{|z| /(\lambda+t)} m_{t, x}(d z)\right\} \geq 0
\end{aligned}
$$

for $C$ large enough.

This implies that, for $\varepsilon>0, u-\varepsilon w$ is a bounded from above subsolution of (15). Indeed, using (17) and (18) yields $g(t, x, u(t, x)-\varepsilon w(t, x)) \geq$ $g(t, x, u(t, x))$ as well as $M(u(t, x+z)-\varepsilon w(t, x+z), u(t, x)-\varepsilon w(t, x)) \geq$ $M(u(t, x+z)-\varepsilon[w(t, x+z)-w(t, x)], u(t, x))$; therefore, given $(p, q, A) \in$ $\mathcal{P}^{2,+} u(t, x)$, we conclude that

$$
\begin{aligned}
& -p+\varepsilon \partial_{t} w-\frac{1}{2} \operatorname{tr} a\left(A-\varepsilon D^{2} w\right)-\langle b, q-\varepsilon D w\rangle-g(t, x, u-\varepsilon w) \\
& -\int M(u(t, x,+z)-\varepsilon w(t, x+z), u(t, x)-\varepsilon w(t, x)) m_{t, x}(d z) \leq 0 .
\end{aligned}
$$

One would prove similarly that $v+\varepsilon w$ is a bounded from below supersolution of (15). Proposition 2 then implies that $u-\varepsilon w \leq v+\varepsilon w$ on $(0, T] \times \mathbb{R}^{N}$ and we conclude that $u \leq v$ after sending $\varepsilon \rightarrow 0$.

Remark 5. - Although this section is not concerned with the question of existence, we shall need later a solution of (15). So we just sketch how to obtain one that satisfies

$$
\lim _{|x| \rightarrow \infty} \sup _{(0, T]}|u(t, x)| e^{-|x| /(\lambda+t)}=0
$$

under the hypotheses of Proposition 4 and the additional one:

$$
\lim _{|x| \rightarrow \infty} \sup _{(0, T]}|g(t, x, 0)| e^{-|x| /(\lambda+t)}=0 \text { and } \lim _{|x| \rightarrow \infty}\left|u_{T}(x)\right| e^{-|x| /(\lambda+T)}=0 .
$$

We suppose, without loss of generality, that $g$ and $M$ satisfy (17) and (18) respectively and redefine $C$ so that the expression within brackets in (30) is greater than 1 (instead of 0 ). For every integer $n \geq 1$, we define $w_{n}(t, x)=C_{n}(T+1-t)+\frac{1}{n} w(t, x)$ and easy computations give

$$
\begin{aligned}
-\partial_{t} w_{n} & -\frac{1}{2} \operatorname{tr} a D^{2} w_{n}-\left\langle b, D w_{n}\right\rangle-g\left(t, x, w_{n}\right) \\
& -\int M\left(w_{n}(t, x+z), w_{n}(t, x)\right) m_{t, x}(d z) \\
& \geq C_{n}+\frac{1}{n} w(t, x)-g(t, x, 0) .
\end{aligned}
$$


In view of the asymptotic behaviour of $g$ and $u_{T}$, one can choose $C_{n}$ so that $g(t, x, 0) \leq C_{n}+\frac{1}{n} w(t, x), u_{T}(x) \leq C_{n}+\frac{1}{n} w(T, x)$ and $w_{n+1} \geq w_{n}+1$ on $(0, T] \times \bar{B}_{n}(0)$. Since, for every $n, w_{n}$ is a supersolution of (15), it is not hard to check that $\bar{v}=\min w_{n}$ is a continuous supersolution and satisfies the first inequality in (29). One constructs similarly a continuous subsolution to (15) satisfying the second inequality in (29). We then invoke Proposition 1 to obtain a solution with the required asymptotic behaviour.

A similar analysis extends to the case when $\sigma$ and $b$ are unbounded but satisfy (27). In addition to (19), we shall assume that, for some $n \in(0, \infty)$,

$t, x \mapsto \int|z|^{n} m_{t, x}(d z)$ is continuous and $\int|z|^{n} m_{t, x}(d z) \leq C\left(1+|x|^{n}\right)$

and require the subsolutions and supersolutions to be compared to satisfy the asymptotic condition:

$$
\limsup _{|x| \rightarrow \infty} \sup _{(0, T]} u(t, x)|x|^{-n} \leq 0, \liminf _{|x| \rightarrow \infty} \inf _{(0, T]} v(t, x)|x|^{-n} \geq 0
$$

respectively (which, as before, implies integrability condition (7) for $u$ and (8) for $v$ ).

Proposition 5. - Let $a, b \in C\left((0, T] \times \mathbb{R}^{N}\right)$ satisfy (16) and (27), $g \in C\left((0, T] \times \mathbb{R}^{N} \times \mathbb{R}\right)$ satisfy (25), $M \in C(\mathbb{R} \times \mathbb{R})$ satisfy (3) and (26), $m$ satisfy (5), (19) and (31), and $u_{T} \in C\left(\mathbb{R}^{N}\right)$. Let $u \in U S C\left((0, T] \times \mathbb{R}^{N}\right)$ be a viscosity subsolution of (15) and $v \in L S C\left((0, T] \times \mathbb{R}^{N}\right)$ be a viscosity supersolution of (15), that satisfy (32) respectively.

Then

$$
u \leq v \text { on }(0, T] \times \mathbb{R}^{N}
$$

Proof. - The proof goes exacly the same way as the one of Proposition 4 and we just have to explain how to modify $w$. We first remark that (19), (31) and Hölder's inequality imply that, for all $p \in(0, n]$, $\int \frac{|z|^{p}}{\left(1+|x|^{2}\right)^{p / 2}} m_{t, x}(d z) \leq C$, which yields

$$
\begin{aligned}
& \int\left(1+|x+z|^{2}\right)^{n / 2} m_{t, x}(d z) \\
& \quad \leq 2^{n / 2}\left(1+|x|^{2}\right)^{n / 2} \int\left(1+\frac{|z|^{2}}{1+|x|^{2}}\right)^{n / 2} m_{t, x}(d z) \\
& \quad \leq C\left(1+|x|^{2}\right)^{n / 2} .
\end{aligned}
$$


Hence, setting $w(t, x)=e^{K(T-t)}\left(1+|x|^{2}\right)^{n / 2}$, we deduce that

$$
\begin{gathered}
-\partial_{t} w-\frac{1}{2} \operatorname{tr} a D^{2} w-\langle b, D w\rangle-C_{M} \int|w(t, x+z)-w(t, x)| m_{t, x}(d z) \\
\geq w(t, x)\left[K-n \frac{\operatorname{tr} a(t, x)}{\left(1+|x|^{2}\right)}\right. \\
\left.\quad-n(n-2) \frac{\langle a(t, x) x, x\rangle}{\left(1+|x|^{2}\right)^{2}}-n \frac{\langle b(t, x), x\rangle}{\left(1+|x|^{2}\right)}-C\right] \geq 0
\end{gathered}
$$

for $K$ large.

Remark 6. - An argument analogous to Remark 5 gives a solution to (15) satisfying $\lim _{|x| \rightarrow \infty} \sup _{(0, T]}|u(t, x)||x|^{-n}=0$, under the assumptions of Proposition 5 and $\lim _{|x| \rightarrow \infty} \sup _{(0, T]}|g(t, x, 0)||x|^{-n}=0$ and $\lim _{|x| \rightarrow \infty}\left|u_{T}(x)\right||x|^{-n}=0$.

\section{APPLICATION TO STOCHASTIC DIFFERENTIAL UTILITY}

In this section, we apply the results of the preceding sections to the stochastic differential utility model due to Duffie and Epstein [4] under mixed Poisson-Brownian information. As in the Dekel-Chew model (see [4, example 5]), we restrict ourselves to $M \in C^{2,1}(\mathbb{R} \times \mathbb{R})$ satisfying (3) and

$$
M_{1}(a, a)=1 \text { and } M(a, a)=0 .
$$

As a matter of fact, the assumption that $M_{1}(a, a)=1$ is just required for the stochastic interpretation but plays no role from the PDE point of view. Besides, that $M(a, a)=0$ is not a restriction since we may replace the functions $M$ and $g$ respectively by

$$
\begin{aligned}
M^{\prime}(a, b) & =M(a, b)-M(b, b), \\
g^{\prime}(t, x, a) & =g(t ; x, a)+M(a, a) m_{t, x}\left(\mathbb{R}^{n}\right) .
\end{aligned}
$$

Before stating precise results, we wish to illustrate briefly how (4) is related to the stochastic differential utility model, which solves a particular forward-backward stochastic differential equation adapted to the filtration generated by a Brownian motion and a jump process (see Pardoux and Peng [9] for the Brownian information case). In order to avoid technicalities and not to make too many restrictive assumptions, we keep this discussion very formal, and refer to $\mathrm{Ma}$ [8] for a rigorous presentation of the stochastic model. Let $W$ be a Brownian motion for 
$t \in[0, T]$ and, given $x \in \mathbb{R}^{N}$, consider $\mu(t, x, A)$ a right-continuous with left-hand limits orthogonal martingale measure (for $A$ a borel set in $\mathbb{R}^{N}$ ) that is independent with the Brownian motion and whose characteristic is given by $\pi(t, x, A)=\int_{0}^{t} \int_{A} m_{s, x}(d z) d s$ - we refer to [6] for a presentation of these notions. Given $(t, x) \in(0, T] \times \mathbb{R}^{N}$, we suppose that there is a unique adapted right-continuous solution (which is a Markov process) of the equation

$$
X_{s}^{t, x}=x+\int_{t}^{s} b\left(r, X_{r-}\right) d r+\int_{t}^{s} \sigma\left(r, X_{r-}\right) d W_{r}+\int_{t}^{s} \int z \mu\left(d r, X_{r-}, d z\right),
$$

for $s \in[t, T]$. If $v$ is a smooth function, Ito's formula reads

$$
\begin{aligned}
d v\left(s, X_{s}\right) & =\langle D v, \sigma d W\rangle+\int v\left(s, X_{s-}+z\right)-v\left(s, X_{s-}\right) \mu\left(d s, X_{s-}, d z\right) \\
& +\left[\partial_{t} v+\langle b, D v\rangle+\frac{1}{2} \operatorname{tr} \sigma \sigma^{T} D^{2} v\right. \\
& \left.+\int\left\{v\left(s, X_{s-}+z\right)-v\left(s, X_{s-}\right)-\langle z, D v\rangle\right\} m_{s, X_{s-}}(d z)\right] d s .
\end{aligned}
$$

On the other hand, the stochastic differential utility solves the backward SDE

$$
U_{s}=U_{T}-\int_{s}^{T} \mu_{r} d r-\int_{s}^{T}\left\langle Z_{r}, d W_{r}\right\rangle-\int_{s}^{T} \int \Gamma_{r}(z) \mu\left(d r, X_{r-}, d z\right),
$$

with $U_{T}=u_{T}\left(X_{T}\right)$ and $\mu_{r}=-g\left(r, X_{r-}, U_{r-}\right)-\frac{1}{2} M_{11}\left(U_{r-}, U_{r-}\right)$ $\left\langle Z_{r}, Z_{r}\right\rangle-\int\left\{M\left(U_{r-}+\Gamma_{r}(z), U_{r-}\right)-\Gamma_{r}(z)\right\} m_{r, X_{r-}}(d z)$ (see [4, section 7], where (33) is used in an essential way). We now apply Ito's formula to $u(t, x)=U_{t}$, which we assume is smooth. Remarking that the Markov property for $X$ and uniqueness yield that $U_{s}=u\left(s, X_{s}^{t, x}\right)$, we first identify $Z_{s}=\sigma\left(s, X_{s-}\right)^{T} D u\left(s, X_{s-}\right)$ and $\Gamma_{s}(z)=u\left(s, X_{s-}+z\right)-u\left(s, X_{s-}\right)$, for $s>t$. Plugging these expressions into $\mu_{s}$ and equating the absolutely continuous terms, we let $s \rightarrow t$ to conclude that $u$ should satisfy

$$
\begin{aligned}
-\partial_{t} u & -\frac{1}{2} \operatorname{tr} a D^{2} u \\
& -\left\langle b-\int z m_{t, x}(d z), D u\right\rangle-g(t, x, u)-\frac{1}{2} M_{11}(u, u)\langle a D u, D u\rangle \\
& -\int M(u(t, x+z), u(t, x)) m_{t, x}(d z)=0 \text { in }(0, T) \times \mathbb{R}^{N} ; \\
u(T, x) & =u_{T}(x) \text { on } \mathbb{R}^{N},
\end{aligned}
$$

which is (4) with $b(t, x)-\int z m_{t, x}(d z)$ instead of $b(t, x)$. 
We now return to the study of equation (4). In order to get rid of the quadratic term $\langle a D u, D u\rangle$, we consider the change of variable used by Duffie and Lions [5]

$$
\Psi(a)=\int_{0}^{a} \exp \left[\int_{0}^{b} M_{11}(c, c) d c\right] d b
$$

which is strictly increasing in $a$. The domain of the inverse mapping of $\Psi$, $\Psi^{-1}$, is denoted by $(\alpha, \beta)-\alpha=\lim _{a \rightarrow-\infty} \Psi(a)$ and $\beta=\lim _{a \rightarrow+\infty} \Psi(a)$. As $\Psi$ is strictly increasing, if $v$ is a subsolution (resp. supersolution) of (4), the function $u=\Psi(v)$ is a subsolution (resp. supersolution) of

$$
\begin{aligned}
-\partial_{t} u & -\frac{1}{2} \operatorname{tr} a D^{2} u-\langle b, D u\rangle-\bar{g}(t, x, u) \\
& -\int \bar{M}(u(t, x+z), u(t, x)) m_{t, x}(d z)=0 \text { in }(0, T) \times \mathbb{R}^{N} \\
u(T, x)= & \Psi\left(u_{T}(x)\right) \text { on } \mathbb{R}^{N},
\end{aligned}
$$

with the notations

$$
\begin{aligned}
\bar{g}(t, x, a) & =g\left(t, x, \Psi^{-1}(a)\right) \Psi^{\prime}\left(\Psi^{-1}(a)\right), \\
\bar{M}(a, b) & =M\left(\Psi^{-1}(a), \Psi^{-1}(b)\right) \Psi^{\prime}\left(\Psi^{-1}(b)\right), \text { for } a, b \in(\alpha, \beta) .
\end{aligned}
$$

In the case of bounded viscosity solutions, an application of Proposition 1 and Proposition 3 readily yields an existence and comparison result. Indeed, we first observe that the functions $\Psi^{-1}$ and $\Psi^{\prime}\left(\Psi^{-1}\right)$ are locally Lipschitz continuous on $(\alpha, \beta)$, hence $\bar{M}$ is locally Lipschitz continuous on $(\alpha, \beta)$. Moreover, the preceding remark, together with (25) and

$$
|g(t, x, a)| \leq C_{R} \text { when } a \in[-R, R]
$$

imply that $\bar{g}$ satisfies (23) (for $a, b \in\left(\alpha_{R}, \beta_{R}\right)$ ).

Theorem 1. - Let $a, b \in C\left((0, T] \times \mathbb{R}^{N}\right)$ satisfy (16), $g \in$ $C\left((0, T] \times \mathbb{R}^{N} \times \mathbb{R}\right)$ satisfy (25) and (35), $M \in C(\mathbb{R} \times \mathbb{R})$ satisfy (3) and (33), $m$ satisfy (5) and (19), and $u_{T} \in C_{b}\left(\mathbb{R}^{N}\right)$.

Comparison. Let $u \in U S C\left((0, T] \times \mathbb{R}^{N}\right)$ be a bounded from above viscosity subsolution of (4) and $v \in L S C\left((0, T] \times \mathbb{R}^{N}\right)$ be a bounded from below viscosity supersolution of (4).

Then

$$
u \leq v \text { on }(0, T] \times \mathbb{R}^{N} .
$$

Existence. There is a unique bounded viscosity solution of (4). 
Proof. - Supposing $\min v \leq \max u$ (or comparison is trivial), we extend $\bar{g}$ and $\bar{M}$ for $a, b \in \mathbb{R}$ by truncation so that they coincide on $[\Psi(\min v), \Psi(\max u)] \subset(\alpha, \beta)$ and satisfy (23) and (24). We then apply Proposition 3 and Remark 3 to obtain comparison. As far as the existence is concerned, one may observe that, for $K$ sufficiently large, $w(t, x)=\exp K(T+1-t)$ is a supersolution of (4) thanks to (25) and (35), while $-w(t, x)$ is a subsolution of (4). Therefore, we may invoke Proposition 1 to obtain a bounded solution of (4).

We now turn to the unbounded case and require that $\bar{g}$ and $\bar{M}$ satisfy (25) and (26) respectively. When $\alpha>-\infty$ or $\beta<\infty$, these regularity assumptions allow to extend $\bar{g}$ and $\bar{M}$ for $a, b \in[\alpha, \beta]$ and then for $a, b \in \mathbb{R} \times \mathbb{R}$ by truncation (with possibly infinite values for $\bar{g}$ ). We keep the notations $\bar{g}$ and $\bar{M}$ for these extensions and we assume that they are well defined and continuous on $(0, T] \times \mathbb{R}^{N} \times \mathbb{R}$ and $\mathbb{R} \times \mathbb{R}$ respectively. In addition we require that, for some $\eta>0$,

$$
\begin{aligned}
& \bar{g}(t, x, a) \leq C(\beta-a) \text { for } a \in[\beta-\eta, \beta] \text { when } \beta<\infty, \\
& \bar{g}(t, x, a) \geq-C(a-\alpha) \text { for } a \in[\alpha, \alpha+\eta] \text { when } \alpha>-\infty .
\end{aligned}
$$

This implies that $\bar{g}(t, x, \beta) \leq 0$ (or equivalently $\lim \sup _{a \rightarrow \infty} g(t, x, a)$ $\Psi^{\prime}(a) \leq 0$ ). In particular, $\beta$ is a supersolution of (34) ( $\alpha$ is a subsolution), which property ensures that a solution with boundary data within $[\alpha, \beta]$ remains in $[\alpha, \beta]$. Our first result concerns the case when $a$ and $b$ are bounded and allows some exponential-like behaviour of the solutions:

$$
\begin{aligned}
& \limsup _{|x| \rightarrow \infty} \sup _{(0, T]} \Psi(u(t, x)) e^{-|x| /(\lambda+t)} \leq 0, \\
& \liminf _{|x| \rightarrow \infty} \inf _{(0, T]} \Psi(v(t, x)) e^{-|x| /(\lambda+t)} \geq 0 .
\end{aligned}
$$

Theorem 2. - Let $a, b \in C_{b}\left((0, T] \times \mathbb{R}^{N}\right)$ satisfy (16), $\bar{g} \in$ $C\left((0, T] \times \mathbb{R}^{N} \times \mathbb{R}\right)$ satisfy (25) and (36), $\bar{M} \in C(\mathbb{R} \times \mathbb{R})$ satisfy (3), (26) and (33), $m$ satisfy (5) and (28), and $u_{T} \in C\left(\mathbb{R}^{N}\right)$.

Comparison. Let $u \in U S C\left((0, T] \times \mathbb{R}^{N}\right)$ be a viscosity subsolution of (4) and $v \in L S C\left((0, T] \times \mathbb{R}^{N}\right)$ be a viscosity supersolution of (4), that satisfy (37) respectively.

Then

$$
u \leq v \text { on }(0, T] \times \mathbb{R}^{N} .
$$

Existence. Assume that

$$
\lim _{|x| \rightarrow \infty} \sup _{(0, T]}|\bar{g}(t, x, 0)| e^{-|x| /(\lambda+t)}=0, \lim _{|x| \rightarrow \infty}\left|\Psi\left(u_{T}(x)\right)\right| e^{-|x| /(\lambda+T)}=0 .
$$


Then, there exists a unique solution of (4) satisfying

$$
\lim _{|x| \rightarrow \infty} \sup _{(0, T]}|\Psi(u(t, x))| e^{-|x| /(\lambda+t)}=0 .
$$

Proof. - Comparison and existence follow respectively from Proposition 4 and Remark 5 when $\alpha=-\infty$ and $\beta=\infty$. In the case $\alpha>-\infty$ or $\beta<\infty$, one combines the arguments of Proposition 3 and Proposition 4 to obtain comparison. Perron's method (Proposition 1) and the fact that $\beta$ is a supersolution ( $\alpha$ is a subsolution) then provide a solution $U \in[\alpha, \beta]$ to (34) - or with the required growth when $\alpha=-\infty$ or $\beta=\infty$. A solution to (4) is then given by $\Psi^{-1}(U)$ provided $U \in(\alpha, \beta)$, to whose proof we now turn.

When $\beta<\infty$, for $\epsilon>0, \nu>0$, we set $w_{\epsilon}(t, x)=\beta-\epsilon e^{C(t-T)}+$ $\epsilon \nu w(t, x)$ with the same $w$ as in the proof of Proposition 4 and the $C$ of assumption (36). Easy computations then prove that $w_{\epsilon}$ is a supersolution to (34) for $\epsilon$ small, $\nu$ arbitrary. Given $x \in \mathbb{R}^{N}$, we choose $\nu$ (depending on $x$ ) so small that $\nu w(t, x)<e^{C(t-T)}$ for all $t \in(0, T]$ and we remark that for some $R>0$ (depending on $x$ ), $\nu w(T, y) \geq 1$ for $y \in B_{R}^{c}(x)$. We then fix $\epsilon>0$ so that $U_{T}(y) \leq \beta-\epsilon$ on $B_{R}(x)$ and use the comparison principle to deduce that $U(t, x) \leq w_{\epsilon}(t, x)$ for all $t \in(0, T]$. By the definition of $\nu$, we conclude that $U(t, x)<\beta$, as claimed. The case $\alpha>-\infty$ is treated similarly.

When $\sigma$ and $b$ have linear growth, we are in the context of Proposition 5 and Remark 6 and we require the subsolutions and supersolutions to satisfy respectively

$$
\limsup _{|x| \rightarrow \infty} \sup _{(0, T]} \Psi(u(t, x))|x|^{-n} \leq 0, \liminf _{|x| \rightarrow \infty} \inf _{(0, T]} \Psi(v(t, x))|x|^{-n} \geq 0 .
$$

Note that our hypotheses on $\sigma$ and $b$ are suited for applications in Finance, where stock prices, which are to determine the utility function, follow a geometrical Brownian motion. With this interpretation in mind, one can choose $m_{t, x}(d z)=\mu \delta_{-x}(z)$, for some $\mu>0$, that models a risk of default of the underlying assets (and which choice fulfils our assumptions).

THEOREM 3. - Let $a, b \in C\left((0, T] \times \mathbb{R}^{N}\right)$ satisfy (16) and (27), $\bar{g} \in C\left((0, T] \times \mathbb{R}^{N} \times \mathbb{R}\right)$ satisfy (25) and (36), $\bar{M} \in C(\mathbb{R} \times \mathbb{R})$ satisfy (3), (26) and (33), $m$ satisfy (5), (19) and (31), and $u_{T} \in C\left(\mathbb{R}^{N}\right)$.

Comparison. Let $u \in U S C\left((0, T] \times \mathbb{R}^{N}\right)$ be a viscosity subsolution of (4) and $v \in L S C\left((0, T] \times \mathbb{R}^{N}\right)$ be a viscosity supersolution of (4), that satisfy (38) respectively.

Then

$$
u \leq v \text { on }(0, T] \times \mathbb{R}^{N} .
$$


Existence. Assume that

$$
\lim _{|x| \rightarrow \infty} \sup _{(0, T]}|\bar{g}(t, x, 0)||x|^{-n}=0, \lim _{|x| \rightarrow \infty}\left|\Psi\left(u_{T}(x)\right)\right||x|^{-n}=0 .
$$

Then, there exists a unique solution of (4) satisfying

$$
\lim _{|x| \rightarrow \infty} \sup _{(0, T]}|\Psi(u(t, x))||x|^{-n}=0 .
$$

\section{REFERENCES}

[1] G. Barles and B. Perthame, Comparison principle for Dirichlet-type Hamilton-Jacobi equations and singular perturbation of degenerated elliptic equations, Appl. Math. Optim., Vol. 21, 1990, pp. 21-44.

[2] A. Bensoussan and J. L. LiONS, Contrôle Impulsionnel et Inéquations Quasi Variationnelles., Dunod, Paris, 1982.

[3] M. G. Crandall, H. Ishil and P. L. Lions, User's guide to viscosity solutions of second order partial differential equations, Bull. Amer. Math. Soc., Vol. 27, 1992, pp. 1-67.

[4] D. DuFfIE and L. EPSTEIN, Stochastic differential utility, Econometrica, Vol. 60, 1992, pp. 353-394.

[5] D. DuFFIE and P. L. Lions, PDE solutions of stochastic differential utility, J. Math. Econ., Vol. 21, 1992, pp. 577-606.

[6] I. I. Ginman and A. V. SковоноD, The Theory of Stochastic Processes, Vol. 3, Springer, Berlin, 1979.

[7] H. IsHII, Perron's method for Hamilton-Jacobi equations, Duke Math. J., Vol. 55, 1987, pp. 369-384.

[8] C. MA, Intertemporal recursive utility in the presence of mixed Poisson-Brownian uncertainty, 1993, Mc Gill University, Montreal, Canada. Working paper series 14.

[9] E. Pardoux and S. G. Peng, Adapted solutions of a backward stochastic differential equation, Systems Control Let., Vol. 14, 1990, pp. 55-61.

[10] A. SAYAH, Equations d'Hamilton-Jacobi du premier ordre avec termes intégro-différentiels, Parties I \& II. Comm P.D.E., Vol. 16, 1991, pp. 1057-1093.

[11] H. M. Soner, Optimal control with state-space constraint II, SIAM J. Control Optim., Vol. 24, 1986, pp. 1110-1122.

[12] H. M. SONER, Optimal control of jump-Markov processes and viscosity solutions, in Stochastic Differential Systems, Stochastic Control Theory and Applications, (W. H. Fleming and P. L. Lions, eds.), IMA Math. Appl., Vol. 10, Springer, Berlin, 1988, pp. 501-511.

(Manuscript received October 18, 1994.)

Vol. 13, $\mathrm{n}^{\circ} 3-1996$. 